\title{
Statistical Power of Fisher Test for the Detection of Short Periodic Gene Expression Profiles
}

\author{
Alan Wee-Chung Liew ${ }^{1^{*}}$, Ngai-Fong Law ${ }^{2}$, Xiao-Qin Cao ${ }^{3}$, and Hong Yan $^{3,4}$ \\ ${ }^{1}$ School of Information \& Communication Technology, Gold Coast Campus, Griffith University, \\ QLD4222, Australia \\ ${ }^{2}$ Centre for Signal Processing, Department of Electronic and Information Engineering, \\ The Hong Kong Polytechnic University, Hong Kong. \\ ${ }^{3}$ Department of Electronic Engineering, City University of Hong Kong, Hong Kong \\ ${ }^{4}$ School of Electronic and Information Engineering, University of Sydney, NSW2006, Australia \\ Email: a.liew@griffith.edu.au, ennflaw@polyu.edu.hk, xiaoqcao@cityu.edu.hk,h.yan@cityu.edu.hk
}

\begin{abstract}
Many cellular processes exhibit periodic behaviors. Hence, one of the important tasks in gene expression data analysis is to detect subset of genes that exhibit cyclicity or periodicity in their gene expression time series profiles. Unfortunately, gene expression time series profiles are usually of very short length, with very few periods, irregularly sampled and are highly contaminated with noise. This makes the detection of periodic profiles a very challenging problem. Recently, a hypothesis testing method based on the Fisher $g$-statistic with correction for multiple testing has been proposed to detect periodic gene expression profiles. However, it was observed that the test is not reliable if the signal length is too short. In this paper, we performed extensive simulation study to investigate the statistical power of the test as a function of noise distribution, signal length, SNR, and the false discovery rate. We have found that the number of periodic profiles can be severely underestimated for short length signal. The findings indicate that caution needs to be exercised when interpreting the test result for very short length signals.
\end{abstract}

Keywords: Gene expression profiles, periodicity detection, Fisher exact test, g-statistic, short signal

\section{INTRODUCTION}

Periodic phenomena are widely studied in biology and there are numerous biological applications where periodicities must be detected from experimental data. Gene expression data from microarray experiments are commonly used to measure the cell-cycle activities. An important task in gene expression data analysis is to detect subset of genes that exhibit cyclicity or periodicity in their gene expression time series profiles. However, this is a challenging problem due to the typically small number of measurements per gene. For example, in the human cancer cells study [1] on http://genome-www.stanford.edu/HumanCellCycle/Hela/, the smallest number of measurements per gene is 9. Moreover, the data can be irregularly sampled and is usually contaminated with high level of noise and missing values.

Recently, several methods for detecting periodic gene expression profiles based on statistical hypothesis testing have been proposed [2-4]. Wichert et al. [2] proposed to use the periodogram-based Fisher g-statistic test to determine whether or not a sequence is periodic. Chen [3] proposed a statistical inference approach, called the C\&G procedure, to effectively detect statistically significant periodically expressed genes based on two statistical hypothesis testing procedures, one of which is the g-statistic. Ahdesmäki et al. [4] proposed a robust spectral estimator for the Fisher g-statistic test that has better performance when the noise deviates from the Gaussian distribution. In our recent work [5], we have proposed a new spectral estimation algorithm for unevenly sampled gene expression data and use the g-

* corresponding author 
statistic to perform a ranking of the genes with respect to their periodicity tendency. We did not attempt to give the number of periodic genes in the gene expression datasets since we have observed that the g-statistic hypothesis testing procedure based on the false discovery rate (FDR) framework has low statistical power for short length signals. This lack of statistical power is also observed in [2] and [4]. Nevertheless, to the best of our knowledge, there is no existing work that attempt to quantify the statistical power (and limitation) of the Fisher g-statistic periodicity test when it is applied in practice. Such a study would be very useful to allow researchers to draw correct conclusion when applying the test.

In this paper, we perform a systematic experimental study of the Fisher g-statistic periodicity test to investigate the accuracy of the test with respect to underlying noise distribution, signal length, signal-to-noise ratio (SNR), and chosen level of statistical significance using the FDR. This is done by performing extensive simulation experiments with simulated signals that model the actual time series profiles that arise from microarray gene expression data. This paper is organized as follows. In Section 2, we give an overview of two cellular processes that would give rise to periodic behavior in gene expression data. In Section 3, we give the definition for the Fisher exact test of periodicity. This is followed by Section 4 which contains extensive experimental results to investigate the power of the test with respect to different signal properties. Finally, we conclude the paper by summarizing our findings and their implications for practical applications.

\section{GENE EXPRESSION TIME SERIES PROFILES}

Oscillations arise in genetic and metabolic networks as a result of various modes of cellular regulation. These rhythmic processes occur at all levels of biological organization with widely varying periods, i.e., from fractions of seconds to decades [6]. Examples of biological rhythms include cell division $[7,8,9]$ and circadian rhythms [10, 11].

The cell-division cycle is fundamental to the proliferation of all organisms. In mitotic cell division, a single cell goes through a sequence of events yields two identical daughter cells. Four phases are usually distinguished [12]. In the presynthetic G1 phase (Gap 1), the cell prepares itself for subsequent DNA synthesis. Enzymes and proteins required for initiating and carrying out DNA synthesis are synthesized late in the G1 phase and early in S phase. The G1 phase is followed by the $S$ phase (synthetic phase) in which the cell replicates its DNA. The postsynthetic G2 phase (Gap 2) is the phase after completion of DNA synthesis in which the cells controls whether DNA replication has been completed and prepares for cell division by synthesizing molecules required in mitotic operation. The $\mathrm{M}$ phase (mitotic phase) following the G2 phase is characterized by the disappearance of nuclear membranes and nucleoli, appearance of the spindle apparatus (prophase), condensation of chromatin into chromosomes, parallel alignment of chromosomes in the equatorial plane of the spindle (metaphase), separation of chromosomes into pairs, and simultaneous movement of chromosomes to opposite poles (anaphase), the reassembly of two nuclei (telophase), and cytoplasmic division (cytokinesis), i.e., the segmentation and separation of the cytoplasm, resulting in the formation of two separate cells.

Circadian clocks have been found in virtually all light-sensitive organisms from cyanobacteria to humans. The basic oscillator mechanisms in all of these organisms are thought to rely on negative feedback loops in gene expression similar to the one first postulated in Drosophila [13]. Briefly, the clockwork contains a positive limb, composed of genes encoding transcriptional activators, and a negative limb, made up of genes specifying transcriptional repressors [11]. The expression of the negative limb members is activated by members of the positive limb, and once the nuclear concentration of repressor proteins has reached a critical threshold value, they extinguish the transcription of their own genes. As a consequence, the 
levels of the repressors decrease below the threshold value required for auto-repression, and a new accumulation cycle can ensue.

All these rhythmic cellular processes are regulated by different gene products. Rhythmic cellular processes can be studied by measuring the gene expression profiles through a series of DNA microarray experiments. If the expression patterns of a group of genes are measured over a number of time points, we obtain a time series data describing the rhythmic behaviors of the genes under study.

A well known set of gene expression time series datasets is that of the Yeast Saccharomyces cerevisiae from Spellman et al. [8]. In this set of datasets, the genome-wide mRNA levels for 6178 yeast ORFs are monitored simultaneously using several different methods of synchronization including an alpha-factor-mediated G1 arrest which covers approximately two cell-cycle periods with measurements at 7 minute intervals for 119 minutes with a total of 18 time points, a temperature-sensitive cdc15 mutation to induce a reversible M-phase arrest (24 time points taken every 10 minutes covering approximately 3.5 cell-cycle periods), and a temperature-sensitive cdc28 mutation to arrest cells in G1 phase reversibly (17 time points taken every 10 minutes covering approximately 2 cell-cycle periods), and finally, an elutriation synchronization to produce the elutriation dataset of 14 time points taken every 30 minutes covering approximately 1 cell-cycle period. The left and right panels of Fig. 1 show some example profiles from these 4 datasets that exhibit highly periodic and random behaviors, respectively. Although periodicity can readily be observed in the left panel of Fig.1, in many cases the distinction between periodic and random profiles cannot be easily made.

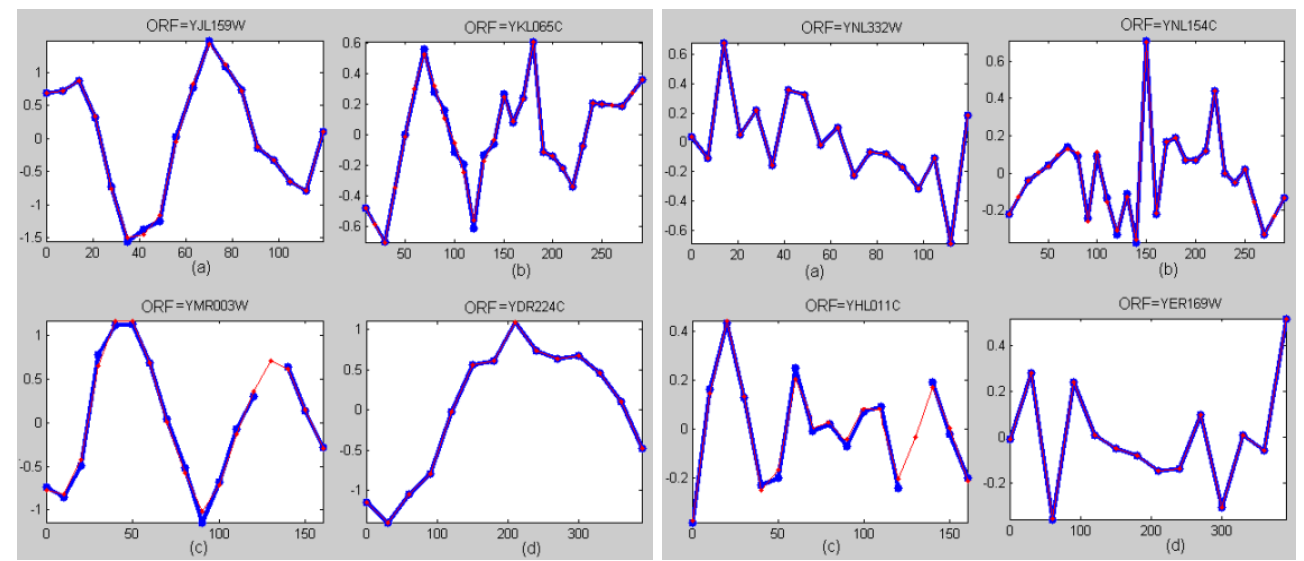

FIG. 1. Left: highly periodic expression profiles, and Right: random profiles from Yeast datasets of Spellman et al. [8]. Profiles (a), (b), (c), (d) correspond to the alpha, cdc15, cdc28, elutriation datasets, respectively. The thin curves in the figure are the interpolated profiles with missing values filled in (see Ref. [5]).

Although periodicity detection in time series data has been well studied in the past, gene expression time series data are different from many traditional time series data in several aspects. First, gene expression time series profile usually contains very few time points. It is not uncommon to see expression profiles that are less than ten time points long. Second, the number of cycles within a profile is usually very few. For example, the 14 time point elutriation dataset of [8] contains only 1 cell-cycle. Third, gene expression data may contain missing values, which need to be estimated from the dataset in advance [14]. Fourth, the time points need not be spaced at regular interval, giving rise to the problem of detecting periodicity in irregularly sampled time series data [5]. Finally, gene expression data is notoriously noisy. All the above makes the detection of periodic gene expression time series profiles an extremely challenging problem and conventional signal processing methods such as the fast Fourier transform (FFT) based techniques do not perform adequately. Instead, 
some authors have recently resorted to statistical hypothesis testing to detect the presence of periodicity in a time series profile [2-4]. One such powerful test is the Fisher g-statistic [15].

\section{FISHER EXACT TEST OF PERIODICITY}

The problem of deciding whether a time series is random or periodic can be cast as a statistical decision problem using hypothesis testing. Wichert et al. [2] proposed to use the Fisher g-statistic to detect periodic sequence. The test determines whether a peak in the periodogram is significant or not. The test proceeds as follows. Given a time series $y(n)$ of length $N$, the periodogram $I(\omega)$ is first computed as

$$
I(\omega)=\frac{1}{N}\left|\sum_{n=1}^{N} y(n) e^{-j \omega n}\right|^{2}, \quad \omega \in[0, \pi]
$$

Furthermore, the periodogram is evaluated at the discrete normalized frequencies

$$
\omega_{l}=\frac{2 \pi}{N}, \quad l=0,1, \ldots, a
$$

where $a=[(N-1) / 2]$ and $[x]$ denotes the integer part of $x$. If a time series has a significant sinusoidal component with frequency $\omega_{k}$, then the periodogram will exhibit a peak at that frequency $\omega_{k}$. Fisher derived an exact test of the significance of the spectral peak by introducing the Fisher $g$-statistic $[15,16]$ defined as,

$$
g=\frac{\max _{l} I\left(\omega_{l}\right)}{\sum_{l=1}^{a} I\left(\omega_{l}\right)}
$$

In Fisher's test, one is testing the null hypothesis $H_{0}$ that the spectral peak is statistically insignificant against the alternative hypothesis $H_{1}$ that there is a periodic component in the signal $y(n)$. Under the Gaussian noise assumption, the exact distribution of the $g$-statistic under the null hypothesis $H_{0}$ is given by

$$
P(g>x)=\sum_{k=1}^{b}(-1)^{k-1} \frac{a !}{k !(a-k) !}(1-k x)^{a-1}
$$

where $b$ is the largest integer less than $1 / x$ and $x$ is the observed value of the g-statistic. Equation (4) yields a p-value that allows to test whether a given time series behaves like a random sequence. Large value of $g$ indicates a strong periodic component and leads us to reject the null hypothesis.

Since there are multiple gene expression profiles to be tested, there is a possibility that a profile can have a small p-value by chance even though it is a random sequence. To correct for multiple testing, Wichert et al. used the method of False Discovery Rate (FDR) [17] to control the expected proportion of false positives at a given rate $q$. The FDR procedure for the set of p-values arranged in ascending order $p_{(1)}, p_{(2)}, \ldots, p_{(G)}$ with corresponding genes

$g_{(1)}, g_{(2)}, \ldots, g_{(G)}$ is as follows:

1. Let $i_{q}$ be the largest $i$ for which $p_{(i)} \leq \frac{i}{G} q$, 
2. then reject the null hypothesis for all genes $g_{(1)}, g_{(2)}, \ldots, g_{\left(i_{q}\right)}$

Although the exact distribution of the Fisher $g$-statistic is available analytically, our experience is that care must be taken when applying it in practice. In particular, we observed that the statistical power of the test is poor when the time series signal is very short. To quantify our observation with respect to different signal characteristics, we perform a series of experiments with simulated signals as reported in the next section.

\section{STATISTICAL POWER OF TEST}

\section{Simulated signals}

To investigate the statistical power of the Fisher exact test, we perform the test for dataset of simulated signals. The simulated signal is given by

$$
y(n)=A \cos (2 \pi \mathrm{n} / \mathrm{T}+\phi)+\varepsilon(n)
$$

where $T$ is the period, $A$ is the amplitude of the sinusoid, $\phi \in[-\pi, \pi]$ is the phase, $n=1, \ldots, N$, and $\varepsilon(n)$ is Gaussian noise sequence of zero mean and unit variance. This signal model is frequently used to model gene expression profiles that exhibit cyclic behavior. Since the periodogram is invariant to $\phi$, we set $\phi$ to zero for the simulated signals. For Spellman's Yeast cell-cycle datasets [8], the number of time points per period ranges between 8 to 14 and the number of periods covered by the gene expression profiles ranges from 1 to 4 . This characteristic is taken into consideration in our simulation experiments.

\section{Empirical versus Exact Distribution}

Fisher derived the exact distribution in (4) for the $g$-statistic computed from the periodogram defined in (1). To see whether the empirically calculated probability density function of the $g$ statistic agrees with the exact distribution given by (4), we generated a test dataset consisted of 10000 random signals using (5), where $A$ is set to zero. Multiple test datasets are generated for random signals of length $N=10$ to 100 with an increment of 5 . The maximum absolute error between the empirically obtained $\hat{P}(g>x)$ and the exact distribution of (4) is shown in Fig.2a. For each signal length, 30 simulation runs are performed and the mean $(\mu)$ \pm 2 standard deviations ( $\sigma$ ) are plotted. We see that for short length signals $(N<40)$, the deviation from the exact distribution tends to be significant. Fig.2b shows the exact distribution and the empirical distribution for $N=10$. The deviation from exact distribution can be clearly seen. For larger value of $N$ (i.e. $N>40$ ), no significant deviation can be observed from the visual plot.

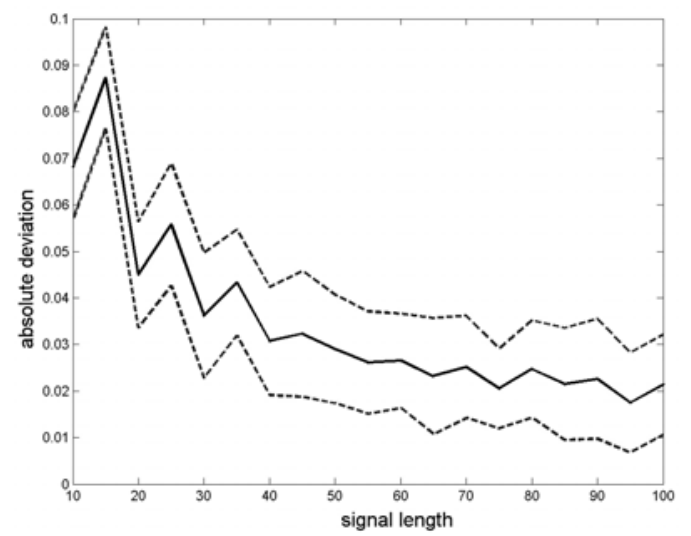

(a) 


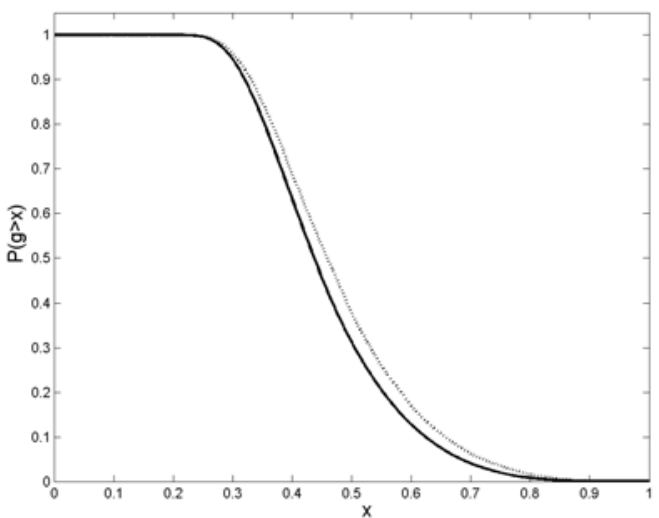

(b)

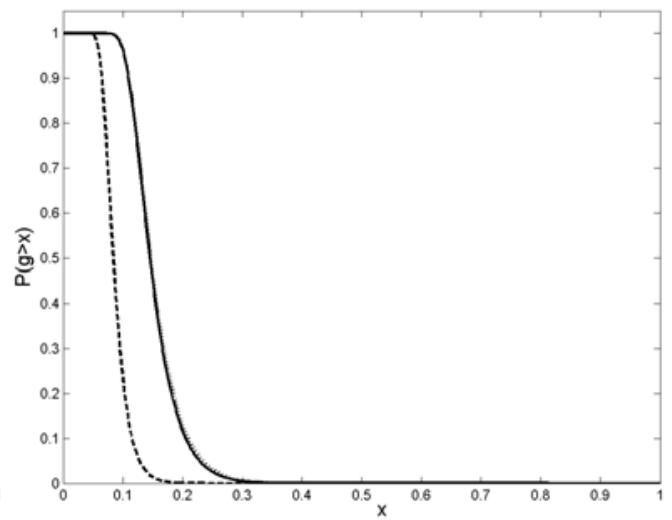

(c)

FIG. 2. (a) The maximum absolute error between the empirically obtained distribution and the Fisher exact distribution. The dashed curves are $\pm 2 \sigma$ around the mean $\mu$ (solid curve). (b) Empirically computed distribution (dashed curve) versus theoretical distribution (solid curve) for short length signal $(N=10)$. (c) For $N=$ 50, the empirical distribution (dotted line) obtained using the periodogram of (1) and the exact distribution (solid curve) is almost indistinguishable. If the robust spectral estimator of Ref. [4] is used, the empirical distribution (dashed curve) can deviate significantly from the exact distribution (solid curve).

Although good agreement is obtained between the empirical and exact distribution for large $N$, we also notice that if a different method of calculating a signal's power spectrum is used, the deviation from the exact distribution can be very significant. Fig.2c shows the exact distribution, the empirical distribution using the periodogram of (1), and using the robust spectral estimator proposed in [4] for $N=50$. We see that for the case where the robust spectral estimator is used, the empirical distribution deviates significantly from the exact distribution. Hence, in the situation that $N$ is small or when a different spectral estimator is used, it is necessary that an empirically computed null distribution obtained from a large number of simulated random signals is used in place of the exact distribution during hypothesis testing. In the case of a real dataset, the dataset of random signals can be obtained by random permutation of the time points of each signal to destroy any periodicity in the signal.

\section{Deviation from Gaussianity Assumption}

Fisher derived the exact distribution of the $g$-statistic for the null hypothesis in (4) under the Gaussian noise assumption. However, in many practical situations the noise distribution is usually not known or may not be Gaussian. To test whether the non-Gaussianity affects the test, we generated a test dataset consisted of 10000 random signals with lognormal distribution. The lognormal distribution is chosen since there is evidence that the noise in microarray gene expression data is lognormal distributed [18]. Using (5), the signal length $N$ is set to 30,50 and 70, $A$ is set to zero, and $\varepsilon(n)$ is a lognormal distributed noise sequence with parameters $\mu=0$ and $\sigma=1$. The mean and variance of the lognormal distribution is then given by $\mu=e^{\mu+0.5 \sigma^{2}}$ and $\operatorname{var}=\left(e^{\sigma^{2}}-1\right) e^{2 \mu+\sigma^{2}}$, respectively. Fig. 3 shows a plot of empirical distribution and the exact distribution for $N=30,50$, and 70 . It can be seen that there is noticeable deviation from the exact distribution when the noise signal is lognormal distributed, although the deviation is much smaller than when a different spectral estimator is used (compare Fig.3 to Fig.2c). The maximum absolute (sum of absolute) deviations are 0.12 (35.86), 0.14 (27.68), 0.15 (21.90) for the three sets of 10000 lognormal distributed random signals of length $N=30,50$, and 70, respectively. The experimental results seem to suggest that there is some effect on the Fisher statistical test when the underlying noise distribution is not Gaussian. Fortunately, at least for the lognormal case, the deviation is not too severe. 

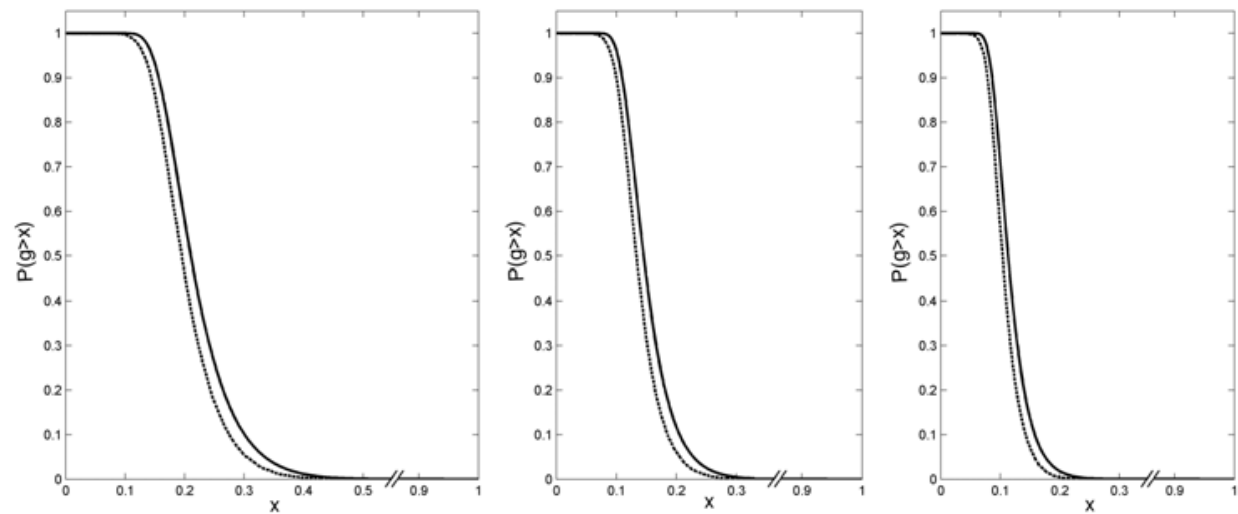

FIG. 3. For $N=30,50$ and 70 (left to right), the empirical distribution computed from lognormal distributed random signals deviates from the exact distribution.

\section{Experimental Setup}

For each of the experiments describe below, each test dataset consists of 4000 random signals and 1000 periodic signals (i.e. $20 \%$ of total number of signals) embedded in zero mean unit variance iid Gaussian noise. Due to the large deviation from the exact distribution for very short signal length, we resort to empirically generate the null distribution by performing random permutation of the time points of each signal in the test set. Periodicity is then determined by hypothesis testing using the empirical distribution. To correct for multiple testing, the FDR framework as described in [2] is adopted. For each simulation, three quantities are determined: (1) the number of periodic signals detected by the test $(L),(2)$ the number of true positives (TP), and (3) the number of true positives in the top 1000 signals $(Z)$ as ranked by their p-values.

\section{Power of Fisher Test versus Signal Length}

For this experiment, we investigate the effect of signal length on the power of the Fisher test. We set $T=10, A=\sqrt{2}$ (giving a SNR of $0 \mathrm{~dB}$ ), and the number of signal time points $N$ is varied from 10 to 100 (in step of 5) giving a signal spanning from 1 to 10 periods. The false discovery rate is set to FDR $=0.05$. For each $N$, we performed 30 simulation runs and calculated $\mu$ and $\sigma$. The results for $L, T P$, and $Z$ versus $N$ are plotted in Fig. 4 . We see that the test is poor if signal length is short and/or signal is not integer number of periods. If the signal length is an integer number of periods, the test is good when $N>40$. The poor result for signal length with non-integer number of periods is due to the truncation effect (windowing) of finite length signal when computing the periodogram using (1). This truncation effect is still very significant when $N>40$. This has important implication when testing for periodicity in real signals of short finite length with unknown period - severe underestimation can occur. The $T P$ versus $N$ plot of Fig. 4(b) is very similar to Fig. 4(a), indicating that most of the signals detected as periodic are indeed periodic. If we count the number of true positives within the top 1000 signals ranked by the $g$-statistics (or equivalently the p-values), we can see from Fig.4(c) that even when the test fails, the ranking still returns many of the periodic signals (i.e., at $N=10$ and at $N=20$ ). This ranking has been exploited by us for periodicity detection in gene expression time series data in a recent paper where the Fisher test cannot be reliably performed [5]. 


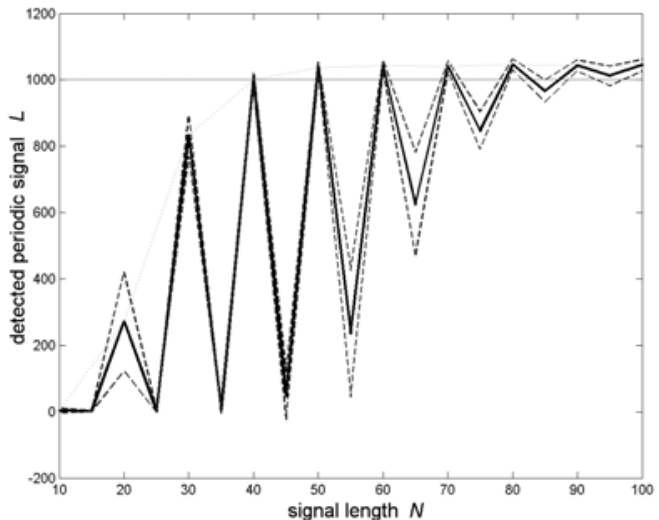

(a)

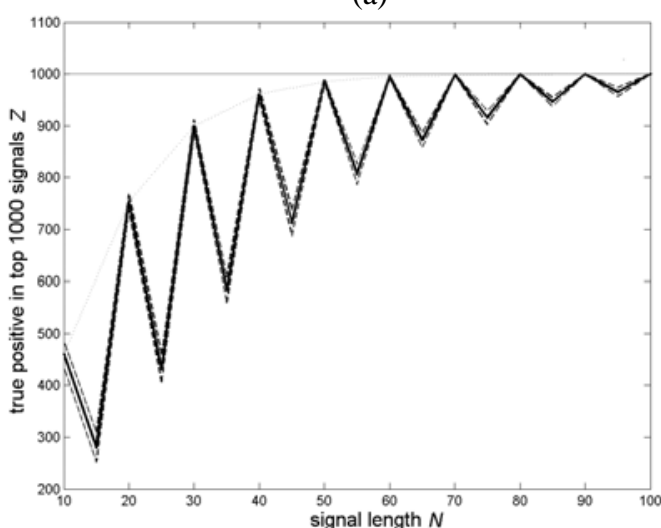

(c)

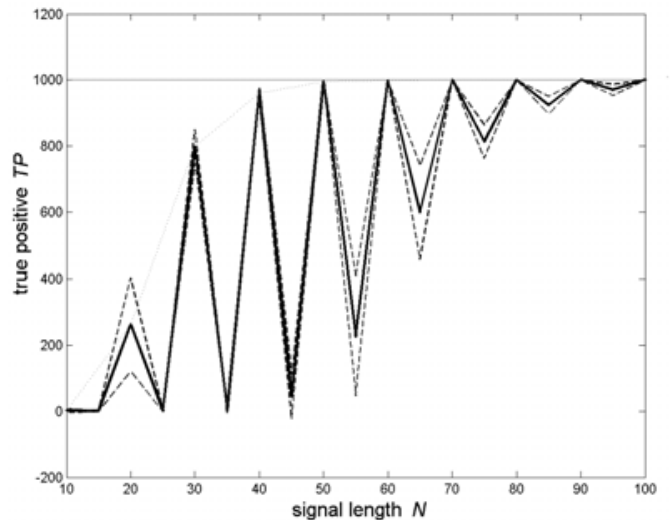

(b)

FIG. 4. (a) Detected periodic signals as a function of signal length $N$. (b) The number of true positives in the detected periodic signals as a function of $N$. (c) The number of true positives in the top 1000 ranking as a function of $N$. In each plots, the dashed curves are $\pm 2 \sigma$ around the mean $\mu$ (solid curve).

In Table 1, we tabulate the mean value of $L$, the mean value of $T P$, and the mean value of $Z$, for signal length with integer number of periods taken from the graphs in Fig. 4. It can be seen that the power of the test deteriorates significantly when signal length is short. A signal length of around 40 or longer is needed to produce a good result when testing for periodicity using the Fisher test.

TABLE 1: The mean value of $L, T P$, and $Z$ as a function of $N$ taken from Fig.4

\begin{tabular}{l|llllllllll}
\hline $\begin{array}{l}\text { signal } \\
\text { length }\end{array}$ & 10 & 20 & 30 & 40 & 50 & 60 & 70 & 80 & 90 & 100 \\
\hline L & 4 & 271 & 832 & 1001 & 1037 & 1043 & 1042 & 1046 & 1044 & 1044 \\
TP & 2 & 262 & 798 & 960 & 994 & 999 & 1000 & 1000 & 1000 & 1000 \\
Z & 461 & 754 & 899 & 961 & 985 & 995 & 998 & 999 & 1000 & 1000 \\
\hline
\end{tabular}

We also investigate whether the power of the test is affected by the sampling rate of the underlying sinusoid. For this we set the signal length $N=60$, but vary the period as $T=\{10,20,30\}$. The other parameters are: $A=\sqrt{2}$, FDR $=0.05$. We perform 30 simulation runs for each $T$, with each dataset consists of 4000 random signals and 1000 periodic signals embedded in Gaussian noise. The results are tabulated in Table 2. We see that higher sampling rate reduces the variance of the periodicity test. This is intuitively to be expected since the higher the sampling rate, the better a sinusoid can be detected with certainty in the signal. 
TABLE 2: The $\mu \pm \sigma$ for $L$ as a function of signal period $T$ for a signal of length 60

\begin{tabular}{l|lll}
\hline signal period $T$ & 10 & 20 & 30 \\
\hline $\mathrm{L}$ & $1043 \pm 8.88$ & $1043 \pm 8.26$ & $1043 \pm 5.40$ \\
\hline
\end{tabular}

\section{Power of Fisher Test versus SNR}

We consider signals with different SNR ranging from -3dB to $3 \mathrm{~dB}$, with a step of $1 \mathrm{~dB}$. The amplitude $A$ of the signals is set to give the required SNR by using the fact that the signal power of a sinusoid is given by $A^{2} / 2$ and the noise power of zero mean unit variance iid Gaussian noise is 1 . The other parameters of the simulated signals are: $T=10, N=50$. FDR is set to 0.05 . For each SNR, we performed 30 simulation runs and calculated $\mu$ and $\sigma$. The results for $L, T P$, and $Z$ versus SNR are plotted in Fig. 5 . We see that the power of the test improves with higher SNR. For this experiment, a SNR of $0 \mathrm{~dB}$ (i.e., $A=\sqrt{2}$ ) or higher gives very good result. However, even for very noisy signals (with SNR as low as -3dB), the power of the test is still quite high. For SNR $=-3 \mathrm{~dB}$, the test was able to detect 748 periodic signals, of which 715 are true positives.

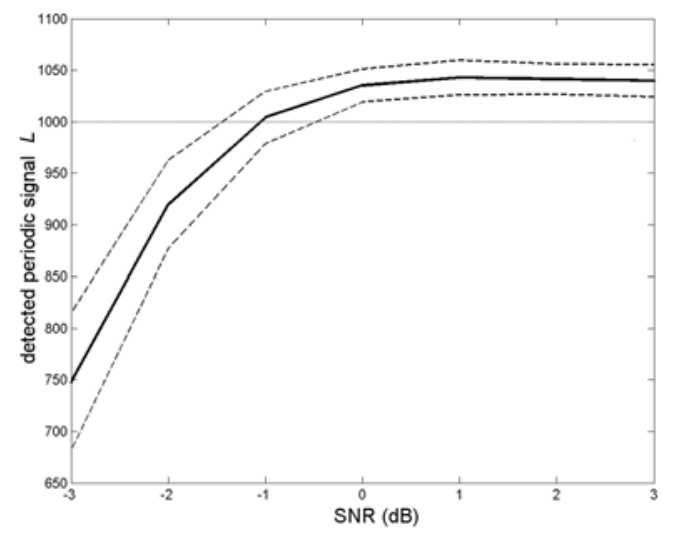

(a)

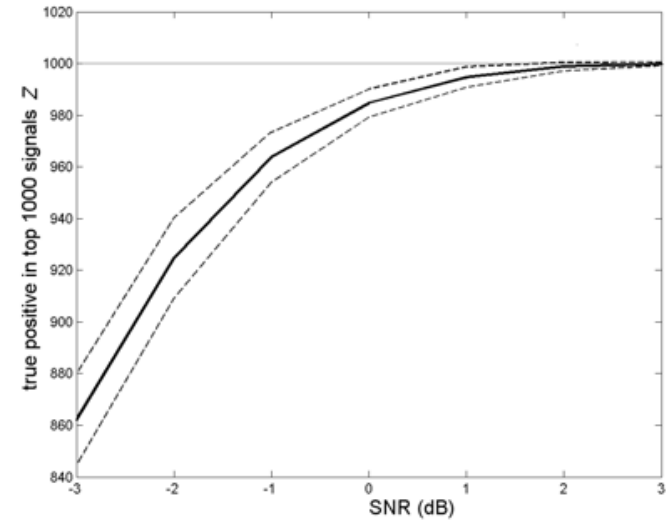

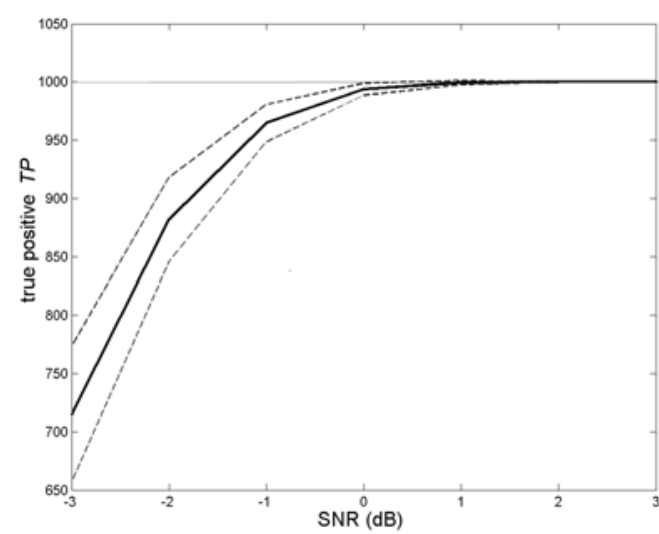

(b)

(c)

FIG. 5. (a) Detected periodic signals as a function of SNR. (b) The number of true positives in the detected periodic signals as a function of SNR. (c) The number of true positives in the top 1000 ranking as a function of SNR. In each plots, the dashed curves are $\pm 2 \sigma$ around the mean $\mu$ (solid curve).

\section{Power of Fisher Test versus FDR}

In this experiment, we investigate the power of the test when different FDR are used. The signal parameters are: $T=10, A=\sqrt{2}, N=50$. For each FDR, we performed 30 simulation runs and calculated $\mu$ and $\sigma$. The results for $L, T P$, and $Z$ versus FDR are plotted in Fig. 6. 
As FDR increases, the number of periodic signals detected $(L)$ also increases as expected, since the number of false positives increases when FDR increases. However, looking at Figs. 6(a) and 6(b), we see that at FDR beyond $0.04 \sim 0.05$, most of the increase in $L$ is due to the increase in false positives. Note that since $Z$ is independent of FDR, the plot in Fig. 6(c) shows constant $Z$ for different FDR.

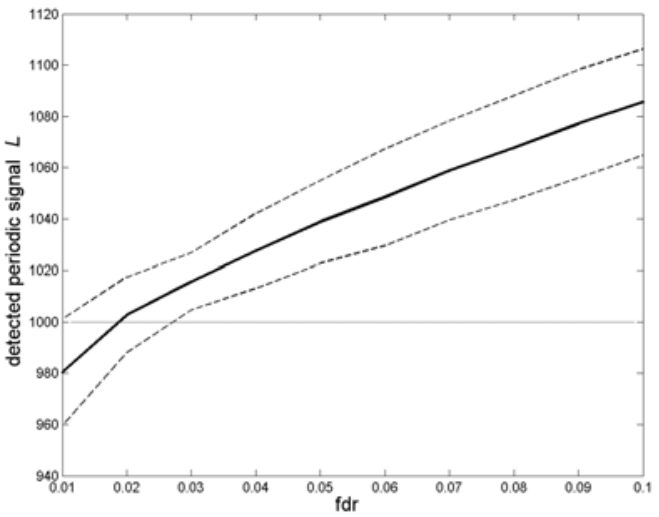

(a)

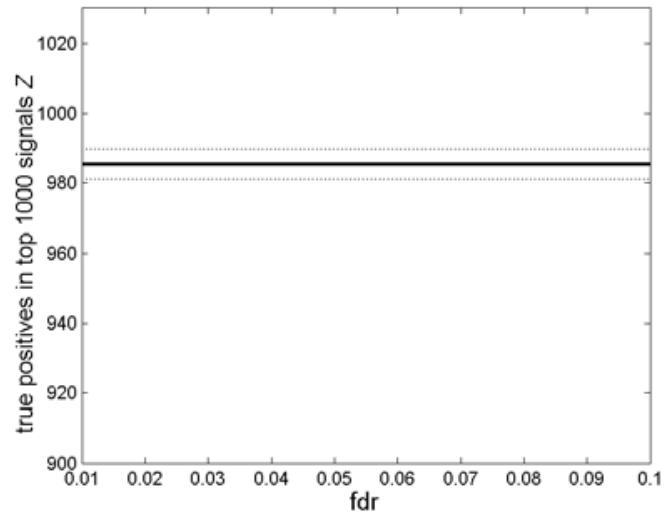

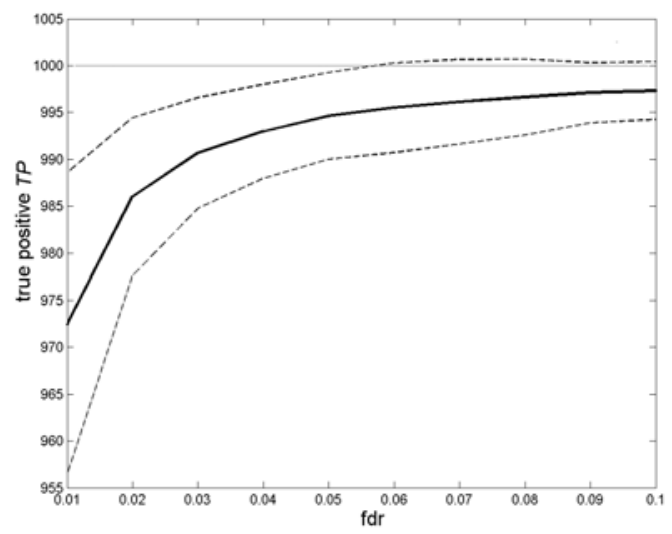

(b)

FIG. 6. (a) Detected periodic signals as a function of FDR. (b) The number of true positives in the detected periodic signals as a function of FDR. (c) The number of true positives in the top 1000 ranking as a function of FDR. In each plots, the dashed curves are $\pm 2 \sigma$ around the mean $\mu$ (solid curve).

\section{CONCLUSIONS}

Due to the difficulty in detecting periodicity in noisy gene expression time series profiles of very short length using conventional signal processing techniques, statistical hypothesis testing has been proposed as a promising tool for this task. In particular, the Fisher exact test for periodicity has been used in several recent papers that attempt to quantify the number of periodic gene expression time series profiles in microarray time series data. In this paper, we investigate the statistical power of the Fisher exact test for periodicity in a finite length signal by extensive simulation experiments. Although the theoretical null distribution was derived by Fisher analytically, we have found that the deviation from it can be significant when signal length is short, i.e. when the signal length is shorter than 40-50 time points. Moreover, when the signal does not cover an integer number of periods, significant drop in the statistical power of the test was observed. In this case, a much longer signal is needed for the test to return reliable result. We have also found that Fisher test is relatively robust to noise. We also investigate how the FDR multiple testing correction strategy affects the number of detected periodic signals. Although the Fisher test may be unreliable for short signal, the Fisher $g$ - 
statistic has been observed to provide a useful ranking of periodic signals. All these findings have important implications for periodic gene expression profiles detection as these profiles are often noisy, of very short length, and often with unknown periodicity. In high likelihood, the number of periodic gene expression profiles can be severely underestimated for short length signal as is found with many of the publicly available gene expression datasets. Hence the result returned by the Fisher test has to be taken with caution.

\section{ACKNOWLEDGMENT}

This work is supported by a grant from the Hong Kong Research Grant Council (Project CityU122607).

\section{REFERENCES}

1. M.L. Whitfield, G. Sherlock, A.J. Saldanha, J.L. Murrary, C.A, Ball, K.E. Alexander, J.C. Matese, C.M. Perou, M.M. Hurt, P.O. Brown, and D. Botstein, "Identification of genes periodically expressed in the human cell cycle and their expression in tumors", Mol. Biol. Cell, 13: 1977-2000, 2002.

2. S. Wichert, K. Fokianos, and K. Strimmer, "Identifying periodically expressed transcripts in microarray time series data”, Bioinformatics, 20: 5-20, 2004.

3. J. Chen, "Identification of significant periodic genes in microarray gene expression data", BMC Bioinformatics, 6: 286-297, 2005.

4. M. Ahdesmäki, H. Lähdesmäki, R. Pearson, H. Huttunen, and O. Yli-Harja, “ Robust detection of periodic time series measured from biological systems”, BMC Bioinformatics, 6:117, 2005.

5. A.W.C. Liew, J. Xian, S. Wu, D. Smith, and H. Yan, "Spectral estimation in unevenly sampled space of periodically expressed microarray time series data”, BMC Bioinformatics, 8:137, 2007.

6. A. Goldbeter, “Computational approaches to cellular rhythms”, Nature, 420: 238-245, 2002.

7. J.M. Mitchison, “Growth during the cell cycle”, International review of cytology, 226: 165-258, 2003.

8. T.S. Spellman, G. Sherlock, M.Q. Zhang, V.R. Iyer, K. Anders, M.B. Eisen, P.O. Brown, D, Botstein, B. Futcher, "Comprehensive identification of cell cycle-regulated genes of the yeast Saccharomyces cerevisia by microarray hybridization”, Mol. Biol. Cell, 9: 3273-3297, 1998.

9. G. Rustici, J. Mata, K. Kivinen, P. Lió, C. J Penkett, G. Burns, J. Hayles, A. Brazma, P. Nurse, and J. Bähler, "Periodic gene expression program of the fission yeast cell cycle", Nature Genetics 36: 809 - 817, 2004.

10. S.K. Crosthwaite, "Circadian clocks and natural antisense RNA”, FEBS Lett., 567: 49-54, 2004

11. U. Schibler, and F. Naef, "Cellular oscillators: rhythmic gene expression and metabolism”, Current Opinion in Cell Biology , 17(2): 223-229, 2005.

12. A. Maton, D. Lahart, J. Hopkins, M.Q. Warner, S. Johnson, J.D. Wright, Cells: Building Blocks of Life, New Jersey: Prentice Hall, 1997.

13. P.E. Hardin, J.C. Hall and M. Rosbash, "Feedback of the Drosophila period gene product on circadian cycling of its messenger RNA levels", Nature 343: 536-540, 1990.

14. X. Gan, A.W.C. Liew, and H. Yan, "Microarray Missing Data Imputation based on a Set Theoretic Framework and Biological Consideration", Nucleic Acids Research, 34(5):1608-1619, 2006, doi:10.1093/nar/gkl047.

15. R.A. Fisher, “Tests of significance in harmonic analysis”, Proc. R. Soc. A, 125: 54-59, 1929. 
16. P.J. Brockwell and R.A. Davis, Time Series: Theory and Methods (2nd Ed.), Springer Verlag, 1991, Chapter 10.

17. Y. Benjamini, and Y. Hochberg, "Controlling the false discovery rate: a practical and powerful approach to multiple testing”, JR Statist. Soc. B, 57: 289-300, 1995.

18. T.V. den Bulcke, K.V. Leemput, B. Naudts, P. van Remortel, H. Ma, A. Verschoren, B. De Moor, and K. Marchal, "SynTReN: a generator of synthetic gene expression data for design and analysis of structure learning algorithms", BMC Bioinformatics, 7:43, 2006, doi:10.1186/1471-2105-7-43

About the Author-ALAN WEE-CHUNG LIEW received his B.Eng. with first class honors in Electrical and Electronic Engineering from the University of Auckland, New Zealand in 1993 and Ph.D. in Electronic Engineering from the University of Tasmania, Australia in 1997. From 1997 to 2004, he worked as a Research Fellow and later a Senior Research Fellow at the Department of Electronic Engineering, City University of Hong Kong. From 2004 to 2007, he was with the Department of Computer Science and Engineering, The Chinese University of Hong Kong as an Assistant Professor. In 2007, he joined the School of Information and Communication Technology, Griffith University as a Senior Lecturer. His current research interests include computer vision, medical imaging, pattern recognition and bioinformatics. He has served as a technical reviewer for a number of international conferences and journals in IEEE Transactions, IEE proceedings, bioinformatics and computational biology. Dr. Liew is a senior member of the Institute of Electrical and Electronic Engineers (IEEE) since 2005.

About the Author-NGAI-FONG LAW received a B.Eng. degree with first-class honors from the University of Auckland, New Zealand, in 1993 and a Ph.D. degree from the University of Tasmania, Australia, in 1997, both in electrical and electronic engineering. She is currently an Assistant Professor in the Electronic and Information Engineering Department, Hong Kong Polytechnic University, Hong Kong. Her research interests include wavelet transform, pattern recognition and bioinformatics.

About the Author-XIAO-QIN CAO received the degree of Bachelor of Science in Macau University of Science and Technology, Macau, PR China, in2006. Currently, she is a Research Assistant in the Department of Electronic Engineering, City University of Hong Kong, Hong Kong, PR China. Her research interest is bioinformatics.

About the Author-HONG YAN received a B.E. degree from Nanking University of Posts and Telecommunications in 1982, an M.S.E. degree from the University of Michigan in 1984, and a Ph.D. degree from Yale University in 1989, all in electrical engineering. In 1982 and 1983 he worked on signal detection and estimation as a graduate student and research assistant at Tsinghua University. From 1986 to 1989 he was a research scientist at General Network Corporation, New Haven, CT, USA, where he worked on design and optimization of computer and telecommunications networks. He joined the University of Sydney in 1989 and became Professor of Imaging Science in 1997. He is currently Professor of Computer Engineering at City University of Hong Kong. His research interests include image processing, pattern recognition and bioinformatics. He is author, co-author or editor of two books and 300 journal and conference papers in these areas. Professor Yan is a fellow of the Institute of Electrical and Electronic Engineers (IEEE), the International Association for Pattern Recognition (IAPR) and the Institution of Engineers, Australia (IEAust) and a member of the International Society for Computational Biology (ISCB). 